\title{
Corrigendum: Spontaneous DNA breakage in single living Escherichia coli cells
}

Jeanine M Pennington \& Susan M Rosenberg

Nat. Genet. 39, 797-802 (2007); published online 27 May; corrected after print 29 August 2007

In the version of this article initially published, our estimate of the rate of formation of spontaneous DNA double-strand breaks (DSBs) in E. coli proportional to DNA content in humans should read that it differs from that of Vilenchik and Knudson (Proc. Natl. Acad. Sci. USA 100, 12871-12876; 2003 ) by fourfold, not "approximately tenfold" (page 800, line 3, and page 800, line 59). We estimated that there are 0.01 DSBs per E. coli genome replication. Because E. coli has approximately $4.7 \times 10^{6}$ bp per genome (Blattner, F.R. et al., Science 277, 1453-1474; 1997), we estimate that approximately $2 \times 10^{-9}$ DSBs per bp are replicated, or about fourfold fewer than the estimate of about $0.8 \times 10^{-8} \mathrm{DSBs}$ per bp replicated in human somatic cells (or 50 DSBs per diploid human genome replication) from Vilenchik and Knudson (Proc. Natl. Acad. Sci. USA 100, 12871-12876; 2003). This would bring the number of DSBs per human genome replication down to approximately 13, if it were proportional to that in E. coli. Our error arose from calculating the human equivalent based on haploid, not diploid, human genome size. This error has been corrected in the HTML and PDF versions of the article.

\section{Corrigendum: Genome-wide analysis of mammalian promoter architecture and evolution}

Piero Carninci, Albin Sandelin, Boris Lenhard, Shintaro Katayama, Kazuro Shimokawa, Jasmina Ponjavic, Colin A M Semple,

Martin S Taylor, Pär G Engström, Martin C Frith, Alistair R R Forrest, Wynand B Alkema, Sin Lam Tan, Charles Plessy, Rimantas Kodzius,

Timothy Ravasi, Takeya Kasukawa, Shiro Fukuda, Mutsumi Kanamori-Katayama, Yayoi Kitazume, Hideya Kawaji, Chikatoshi Kai,

Mari Nakamura, Hideaki Konno, Kenji Nakano, Salim Mottagui-Tabar, Peter Arner, Alessandra Chesi, Stefano Gustincich,

Francesca Persichetti, Harukazu Suzuki, Sean M Grimmond, Christine A Wells, Valerio Orlando, Claes Wahlestedt, Edison T Liu,

Matthias Harbers, Jun Kawai, Vladimir B Bajic, David A Hume \& Yoshihide Hayashizaki

Nat. Genet. 38, 626-635 (2006); published online 28 April 2006; corrected online 5 May 2006; corrected after print 29 August 2007

In the version of this article initially published, two of the smaller bar plots in Figure 1e were mistakenly duplicated. Specifically, the $Z f p 385$ plot is an erroneous copy of the 137774 plot, and the Txndc7 plot is an erroneous copy of the Pik $3 r 5$ plot. See below for the corrected version of the figure. This error does not change the conclusions of the study in any way, as the bar plots are just a few visual examples of more than 5,000 tag clusters, and the correct plots follow the same distribution patterns as the erroneous ones. This error has been corrected in the HTML and PDF versions of the article.

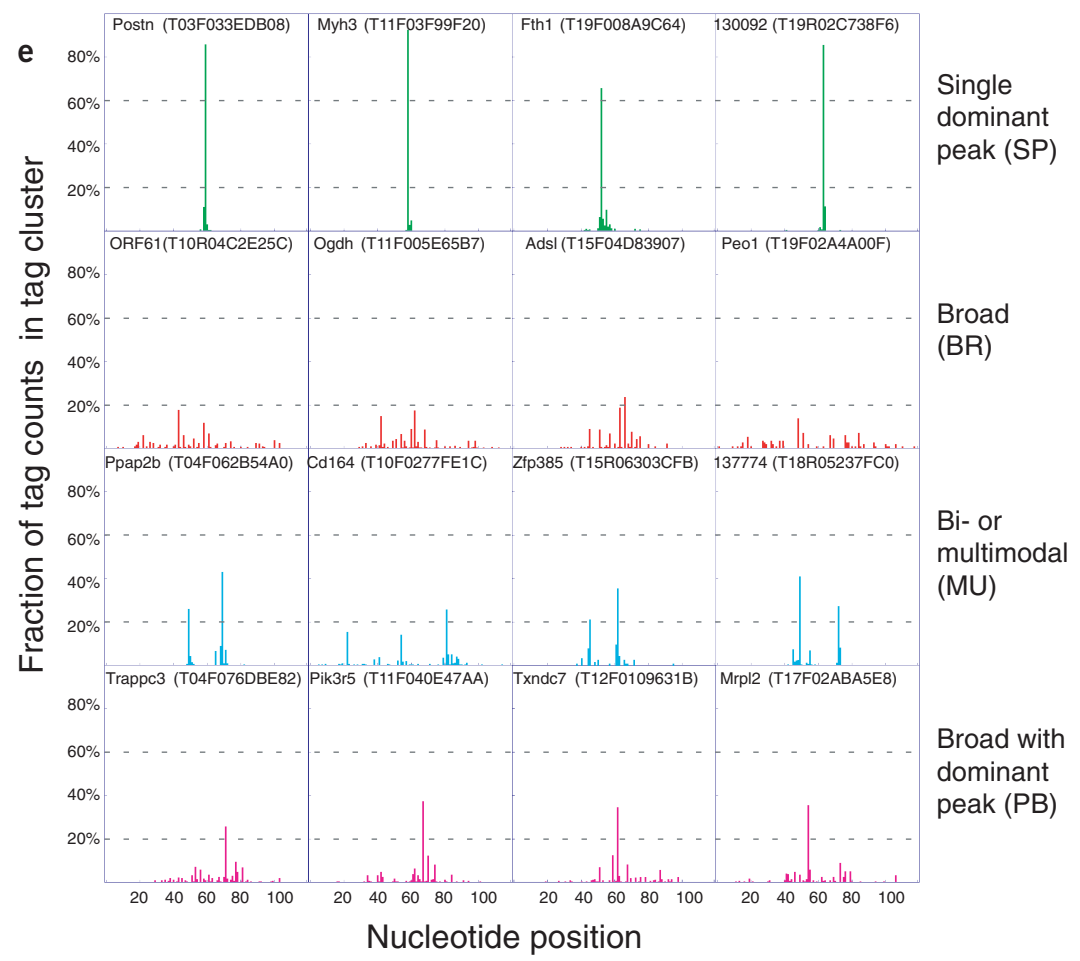

